\title{
A Generic Control System for Transparent Legacy System Migration
}

\author{
A. Adlemo, S.-A. Andréasson \\ Dept. of Computing Science, Chalmers University of Technology \\ S-412 96 Göteborg, Sweden \\ tel. +46-31-772 1043, fax. +46-31-16 5655 \\ adlemo@ce.chalmers.se,andreasson@cs.chalmers.se
}

\section{P. Gullander}

Dept. of Production Engineering, Chalmers University of Technology S-412 96 Göteborg, Sweden

tel. +46-31-772 3616, fax. +46-31-772 3819

perg@pe.chalmers.se

\begin{abstract}
Of importance in defining a balanced automation system (BAS) is product quality. Providing the best possible product quality is paramount for any SME to compete in the global marketplace. To be able to obtain the required product quality, the quality verification should not be left to a human operator solely. Instead, the operator should be backed up by automatic supervision.

This article presents a generic control system whose main activities are to initiate and supervise production. It is illustrated that, part from the main activities, the control system is also capable of providing automated verification of the product quality. Furthermore, as the control system is designed in a modular fashion, it can be used when moving from one BAS to another while the control system is hardly affected, i.e. a transparent legacy system migration.
\end{abstract}

\section{Keywords}

Balanced Automation System, Computer Integrated Manufacturing, Control System, Flexible Manufacturing Systems, Generic Modeling, Legacy System Migration, Object-Oriented Design, Reusability.

\section{INTRODUCTION}

A balanced automation system (BAS) is defined as a system consisting of both automated and manual activities and machines. A BAS is the result of a pre-study to design a manufacturing system, a pre-study influenced by various considerations, e.g. production cost, amount of required flexibility and desired product quality. Furthermore, a BAS is not an invariable 
construction as the manufacturing system environment may change, e.g. the cost of manual labor compared with the introduction of an automatic machine may change. In addition, when moving from one BAS to another, one is confined to the installed legacy manufacturing system. This movement should affect as little as possible of the already installed equipment and programs.

One of the more important features in defining a BAS is product quality. Providing the best possible product quality is paramount for any SME that wishes to compete in the global marketplace. To be able to obtain the required product quality, the quality verification should not be left to a human operator solely. Instead, the operator should be backed up by automatic supervision.

This article presents a generic control system whose main activities are to initiate and supervise production. It is demonstrated that, in addition to these main activities, the control system is also capable of providing automated verification of the product quality. Quality in this case is composed of such things as:

- Verifying the correct ordering of production activities.

- Verifying that the correct product is placed on the correct machine.

- Verifying that no mandatory activity is forgotten.

Verifying that no optional activity is added.

Furthermore, as the control system is designed in a modular fashion, it can be used when migrating from one legacy BAS to another while the control system is hardly affected.

The main theme of the discussions in this article is that, even when human operators perform activities in a manufacturing system that would normally be considered appropriate for automatic control and automatic production, the supervisory portion of the control should not be left to the operators solely if the quality goals of production are to be met. One reason for this is that the equipment needed for supervision (computers, data network and sensors) is relatively cheap as compared with advanced machines, such as a computerized milling machine. To obtain $100 \%$ inspection using humans typically requires a considerable amount of redundancy, often as much as three re-inspections (Dreyfuss, 1989; Freeman, 1988).

\section{CASE STUDY OF A MACHINING CELL}

A production cell for rear axles was installed during 1994 by a truck and bus manufacturer in Sweden. This cell has acted as a case study for the research presented in this article. The production cell is described in more detail in Adlemo et al. (1995a). The case study is used later in this article to describe how the product quality is preserved. The case study is also used to demonstrate a movement from one balanced automation system to another.

The cell consists of seven resources:

A lathe and a multi-operational milling device, together with a quality control station (the producers).

- A gantry crane for loading and unloading the devices (the mover).

- Two output buffers and one input buffer (the locations). 
A local area network connects the resources with one another and a dispatcher. Rear axles are manually entered by the operator at the input buffer. The barcode reader registers incoming axles by identifying their article numbers. The operator can manually enter re-work codes for those axles that have already been through the system but have been rejected by the quality control station. The normal flow through the system for each axle is to visit the milling machine first, then the lathe and, finally, to exit through the normal output buffer. However, the operator can at any time request a specific axle to the quality control station where it is manually tested and compared with its specifications.

\section{A GENERIC CONTROL SYSTEM}

To keep a manufacturing system flexible and to emphasize reusability among applications, a strong separation of the resources and the control system is advocated. The same type of approach is described in Reinaldo Silva et al. (1995). The control system allows any feasible route between the resources, and the route through the system for each type of product is determined in the product routes (Fabian, 1995). The control system would then see that each product is served by the required machining resources in the sequence demanded. This makes the control system reusable between different applications. Only a small application-specific part would have to be re-coded for each specific implementation. Using object-oriented techniques, these parts may be implemented in a general control system as virtual functions that are filled at the implementation stage (Fabian, 1995).

A manufacturing system can be viewed as a finite set of resources, i.e. the machining equipment, shared between a set of users, i.e. the products to be manufactured. For control purposes, we need models of the resources and users. These models are referred to as resource models and operation lists, respectively. For feasible implementation, we want these entities to be as application-unspecific as possible, since this enhances the reusability of the components. Once implemented and thoroughly tested, modules can then be used in similar applications with little or no modification.

The control system described in this article is aimed at completely automatic production. However, the control system could also be used for balanced automation systems that consist of both automated and manual activities and machines.

\subsection{Resources models}

The resource models are based on an explicit mapping of elements of the physical system onto modules of the control system. Using object-oriented analysis and design methods (Shlaer and Mellor, 1992), we create internal resources that correspond to physical devices. For each physical device, there exists a corresponding internal resource that communicates through a proprietary protocol (Figure 1). The internal resources also communicate with other internal resources through handshake messages, and with the dispatcher through control messages (Figure 1).

To increase the modularity and flexibility, the internal resources are separated into a general part and a specific part (Gullander et al., 1995) (Figure 1). The general part and the specific part communicate through internal resource messages. The general part describes a discrete event process, DEP, typically an automaton or a Petri net, representing the behavior of the physical 


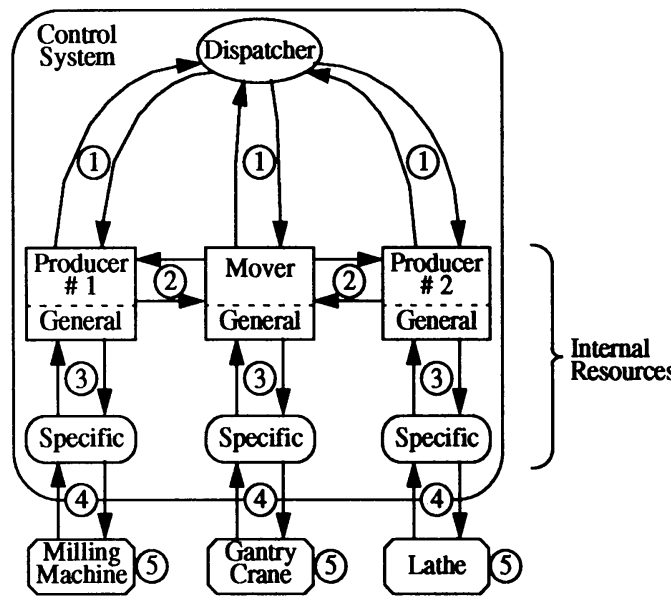

Figure 1 The generic control system structure.

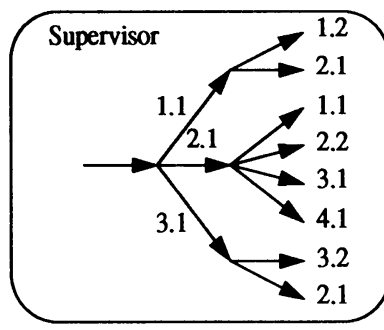

$x . y: x=$ product type

$y=$ operation number

(1) Command messages

(2) Handshake messages

(3) Internal resource messages

(4) Proprietary protocol messages

(5) Physical device code

device (Ramadge and Wonham, 1987). Thus, the general part keeps track of the current state of the physical device. The specific part must translate the high level messages exchanged between the internal resources into some lower level, proprietary protocol that can be understood by the physical device. The general and specific parts communicate via internal resource messages, e.g. MMS messages (MMS, 1990).

\subsection{The supervisor and the dispatcher}

For a manufacturing system to be able to produce, the operation lists must be mapped on the resource model. An operation list describes the steps to be taken in order to produce a specific product on an imaginary production facility. The mapping is one of the main activities necessary to obtain a supervisor (Fabian, 1995). The basic research in this area was done by Ramadge and Wonham (1987). A supervisor is a discrete event process that is used by the dispatcher to execute the work on a manufacturing system that exhibits a pre-specified desired behavior. During execution, the work follows the closed loop system of the supervisor. A description of the automatic synthesis of a supervisor based on operation lists and internal resources is found in Fabian (1995). Barata et al. (1995) describe a similar approach to obtain what they call a controller.

A generic control system structure is illustrated in Figure 1. This control system is a modular system in which the modules communicate through messages. A dispatcher decides what operations shall be carried out a specific product using the supervisor. The dispatcher actively initiates production and movement of products while relying on the production and movement of products being carried out correctly. The dispatcher can be exchanged for a monitor that also uses the supervisor. However, the monitor monitors only production and the movement of products while relying on someone else tom carry out the actual production initiation and movement of products. 


\section{BALANCED AUTOMATION AND AUTOMATION LEVELS}

The production in the machining cell described in section 2 was originally designed to be more or less automatic, i.e. the human interference with production was meant to be kept to a minimum. The only interference was the operator supervising the system. The manufacturing cell illustrates a balanced automation system based on some important input data. However, the machining cell does not necessarily have to consist of completely automated resources, e.g. an SME that does not have the economic resources to buy a gantry crane can exchange it for a fork lift (Adlemo and Andréasson, 1995b; Adlemo and Andréasson, 1996a). This new cell is a balanced automation system as well. Where the exact amount of automation should be placed is governed by a number of concepts, e.g. time, money, safety, flexibility and quality.

This section describes some possible alternatives for automatic production in a manufacturing system, using the manufacturing cell in section 2 as an example. Four main automation alternatives have thus been identified. The list should be read such that each of the automation alternatives, as well as their sub-alternatives, may or may not be included.

\section{Cell control system}

dispatcher / monitor / not present

The control level can exist in three different forms, i.e. dispatcher, monitor or not present. Both the dispatcher and monitor work towards the supervisor with one big difference. The dispatcher, on one hand, actively initiates the production and movement of products while relying on the production and movement of products being carried out correctly. The monitor, on the other hand, monitors only the production and movement of products while relying on someone else to carry out the actual production initiation and movement of products. We strongly suggest that at least the monitor with its activities should always be present, while the rest of the automation alternatives can be chosen more freely.

\section{Mover}

asynchronous material transportation (i.e. transportation between machines)

automatic / manual

synchronous material transportation (i.e. transportation to an assembly machine)

automatic / manual

Asynchronous material transportation. The transportation of material between different machines in a machining cell is asynchronous, i.e. there are no requirements concerning synchronization with other flows of transportation.

In the case study, this automation alternative can be obtained by replacing the expensive gantry crane with a manually operated truck. Instead, here, printed truck orders are needed. If the quality of the products is to be maintained in this case, it is necessary to use sensors at the machines to verify that the correct product is delivered to the correct machine.

Synchronous material transportation. In an assembly cell, as compared with a pure machining cell, there is also a synchronous transportation of material between different stations. The synchronous transportation is special, as the arrival of a piece of material at a station should be coordinated with the arrival of other pieces of material. 


\section{Producer}

automatic / semi-automatic / manual

Another method for reducing costs is to exchange automatically operated machines with semi-automatic or manually operated machines. This can be done for all of the machines or only some of them.

In the case study, this automation alternative can be obtained by replacing the expensive milling machine and the expensive lathe with manually operated equivalents. Instead, here, printed working orders for the machines are needed. If the quality of the products is to be maintained at the same time that automatically operated machines are omitted, it is necessary to use sensors that verify the quality of the products.

\section{Data network}

present / not present

Yet another alternative for reducing costs is to remove the data network. This leaves us with a system with stand-alone machines, where the instructions for the machines must be entered manually as no data network exists to transport instructions.

In the case study, this level can be obtained by removing the local area network that interconnects the machines and the cell controller.

These four different automation alternatives can be combined in a number of possible combinations into what we call automation levels. However, for the product quality to be maintained, the monitor activities should be present (it is implicit that the cell computer is present) (Adlemo and Andréasson, 1995b; Adlemo and Andréasson, 1996a).

\section{TRANSPARENT LEGACY SYSTEM MIGRATION}

As mentioned earlier, a balanced automation system is characterized by an appropriate level of automation and manual work, taking into account such aspects as flexibility and quality. This section briefly describes a generic control system that is capable of accepting the introduction of new machines or new products with a minimum of unnecessary extra work. One of the chief goals in designing the generic control system was to provide flexibility, a flexibility not always found in existing systems, even in so called flexible manufacturing systems where one would expect to find true flexibility. It is illustrated in the following sections how the generic control system also provides a possibility for small companies to migrate, step by step, from manual production to a completely automatic system without having to alter the control system in a major way. As an example, the machining described in section 2 is used.

\subsection{Manual production with manual transportation}

In the first example, the machining cell consists of six resources (for simplicity, the quality control station and the output buffers are not shown). There are three manually operated milling machines (MM1, MM2, MM3), two manually operated lathes (ML1, ML2) and one forklift (T) to perform the work (Figure 2). Apart from these resources there are sensors that verify the correctness of the material that arrives to the manually operated machines. 


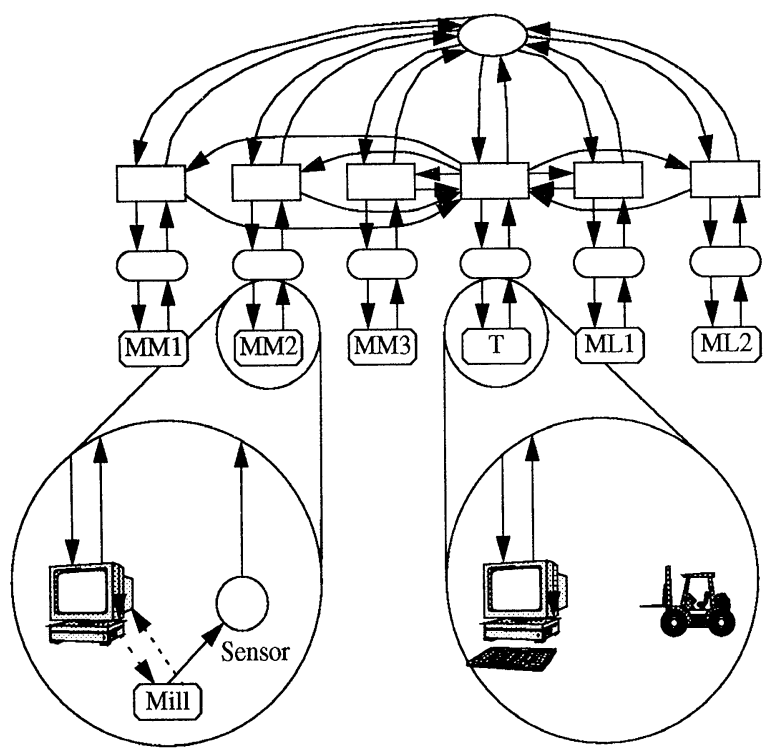

Figure 2 Manual production with manual transportation.

As described in Adlemo and Andréasson (1995b; 1996a), the lowest level of automation that should be accepted in a manufacturing system is the case in which at least a data network and automatic supervision are present. The quality verification should not be left to the human operator solely.

The operator of the cell receives production data via a terminal or on a paper sheet. The data is then carried out by the operator through manual operations on the machines. In a similar manner, the forklift driver is informed by the system where to go next and what to do.

The specific parts of the internal resources are specially designed for the communication between the control system and the manually operated machines (more exactly, the communication between the control system and the terminal or the printer).

\subsection{Semi-automatic production with manual transportation}

In the second example, the machining cell consists of five resources. There are two semiautomatic milling machines (SM1, SM2), two semi-automatic lathes (SL1, SL2) and one forklift (T) to perform the work (Figure 3). Apart from these resources there are sensors that verify the correctness of the material that arrives to the manually operated machines.

The operator of the cell receives production data via a terminal. The data is interpreted by the operator who then carries out the work on the semi-automatic machines. The forklift driver is informed by the system via a terminal where to go next and what to do. The terminal may be operated on board the forklift, accessed from a central location or placed close to the machines. 


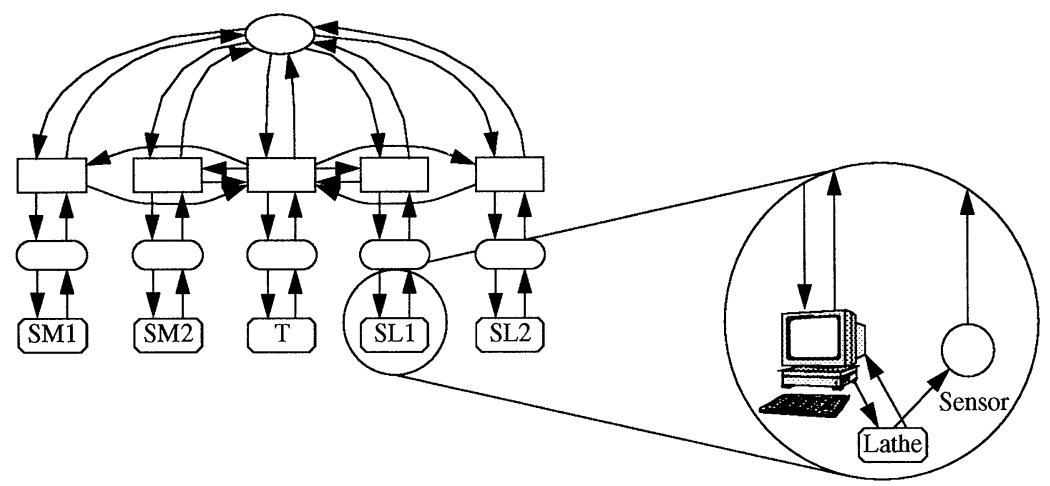

Figure 3 Semi-automatic production with manual transportation.

The specific parts of the internal resources are specially designed for the communication between the control system and the semi-automatic machines. The rest of the control system is intact, without any changes.

If migrating from manual production to semi-automatic production, only the specific parts of the producers must be modified.

\subsection{Automatic production with manual transportation}

In the third example, the machining cell consists of three resources. There are one automatic milling machine (AM1), one automatic lathe (AL1) and one forklift (T) to perform the work (Figure 4).

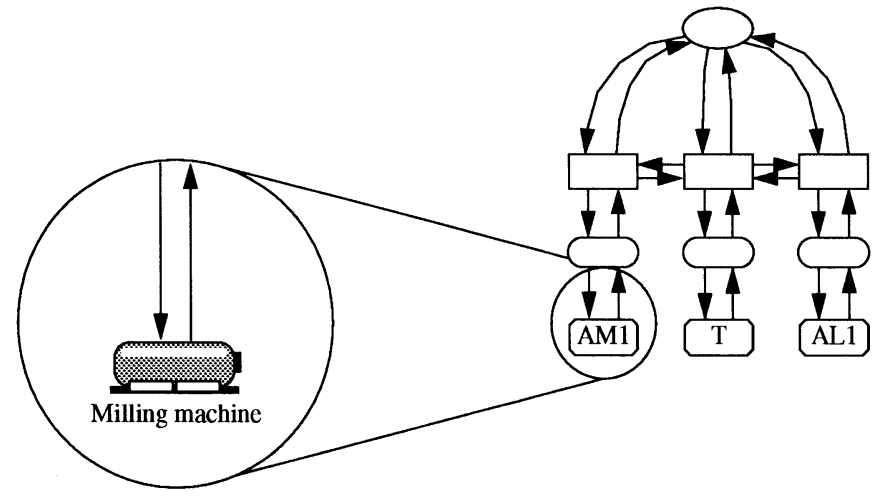

Figure 4 Automatic production with manual transportation. 
The operator of the cell receives production data via a terminal. The data is then transmitted to the semi-automatic machines where the work is carried out. The forklift driver is informed by the system via a terminal as to where to go next and what to do. The terminal may be operated on board the forklift, accessed from a central location or placed close to the machines.

The specific parts of the internal resources are specially designed for the communication between the control system and the automatic machines. The rest of the control system is intact, without any changes.

If migrating from semi-automatic production to automatic production, only the specific parts of the producers must be modified.

\subsection{Automatic production with automatic transportation}

In the fourth example, the machining cell consists of three resources. There are one automatic milling machine (AM1), one automatic lathe (AL1) and one gantry crane (GC) to perform the work (Figure 5).

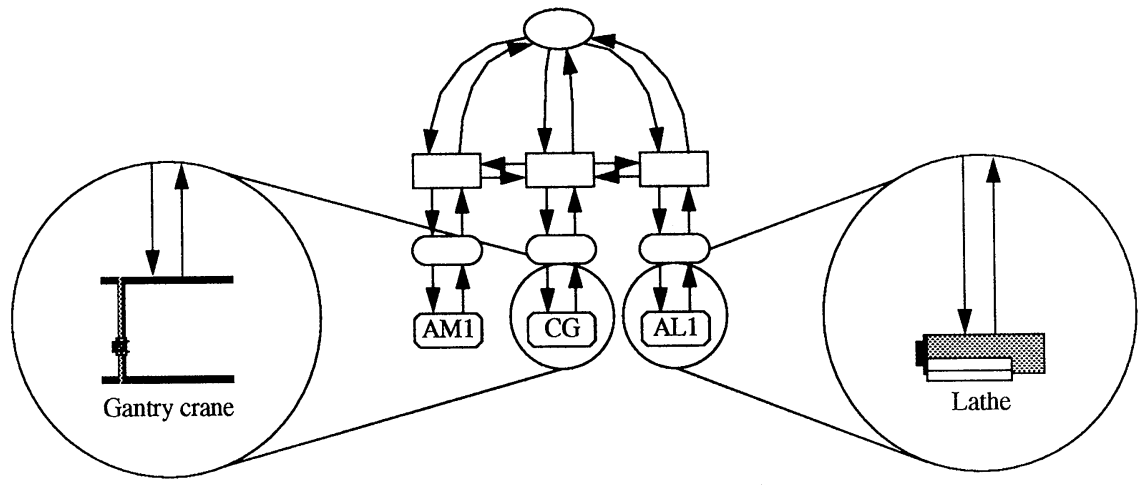

Figure 5 Automatic production with automatic transportation.

The producers and the mover receive production data from the system and carry out the work.

The specific parts of the internal resources are specially designed for the communication between the control system and the automatic machines. The rest of the control system is intact, without any changes.

\subsection{Balanced automation system migration}

The previous sections have described four different combinations of automation alternatives leading to four different balanced automation systems, BASs. All of the four BASs comply with the suggestion that the product quality verification be performed with the help of the control 
system, as can be seen in Table 1 (column 1., Cell control system). All of the BASs have at least a monitor to verify correct production, etc., as described in section 4 .

Table 1 Examples of balanced automation systems
1. Cell control system
D Dispatcher
PA Production Automatic
2. Mover
M Monitor
PS Production Semi-automatic
3. Producer
AA Asynchronous Automatic
AM Asynchronous Manual
PM Production Manual
4. Data network
$P \quad$ Present

\begin{tabular}{lllllll}
\hline & & \multicolumn{3}{c}{ Automation alternatives } \\
\cline { 3 - 7 } No. & Examples of levels of automation & 1. & 2. & 3. & 4. \\
\hline 5.1 & Manual production with manual transportation & M & AM & PM & P \\
5.2 & Semi-automatic production with manual transportation & M & AM & PS & P \\
5.3 & Automatic production with manual transportation & M & AM & PA & P \\
5.4 & Automatic production with automatic transportation & D & AA & PA & P \\
\hline
\end{tabular}

\section{SUMMARY}

In many manufacturing systems, especially in SMEs and in developing countries, it is very important to include human activities early, in the design stage, thus obtaining balanced automation systems. The final outcome when designing a balanced automation system depends on several parameters, of which the product quality achieved by the system at production time is very important to remaining competitive.

This article described a generic control system that implicitly provides for product quality with respect to providing for correct ordering of production, not forgetting any step in production etc. The modularity of the generic control system means that a balanced automation system can be updated when needed to a new balanced automation system, without having to redesign the control system from the very start. The control system thus provides for a transparent legacy system migration.

\section{ACKNOWLEDGEMENTS}

This article was partially funded by the Swedish National Board for Industrial and Technical Development (NUTEK) under grant number 9304792-2. 
Adlemo A., S.-A. Andréasson, M. Fabian, P. Gullander and B. Lennartson (1995a). Towards a truly flexible manufacturing system. Control Engineering Practice, 3(4), 545-54.

Adlemo A. and S.-A. Andréasson (1995b). Balanced automatization levels in manufacturing systems. In Balanced Automation Systems: Architectures and Design Methods, (eds. L. M. Camarinha-Matos and H. Afsarmanesh), Chapman \& Hall, U.K., 393-404.

Adlemo A. and S.-A. Andréasson, (1996a). Balanced automation in flexible manufacturing systems. Submitted to an international journal.

Adlemo A., S.-A. Andréasson, P. Gullander, M. Fabian and B. Lennartson (1996b). Operator control activities in flexible manufacturing systems. Submitted to an international journal.

Barata J., L. M. Camarinha-Matos, W. Colombo and R. Carelli (1995). Integration of objectoriented programming and Petri nets for modelling and supervision of FMS/FAS, in Balanced Automation Systems: Architectures and Design Methods, (eds. L. M. CamarinhaMatos and H. Afsarmanesh), Chapman \& Hall, U.K., 107-20.

Dreyfuss D. D. (1989). Is industry ready for machine vision? - a panel discussion, in Machine Vision for Inspection and Measurement (ed. H. Freeman), Academic Press Inc., New York, U.S.A., 223-36.

Fabian M. (1995). On Object-Oriented Nondeterministic Supervisory Control, Ph. D. thesis, Control Engineering Laboratory, no. 282, Chalmers University of Technology, Sweden (ISBN 91-7197-207-2).

Freeman H. (1988). Machine Vision. Academic Press Inc., Boston, U.S.A.

Gullander P., M. Fabian, S.-A. Andréasson, B. Lennartson and A. Adlemo (1995). Generic resource models and a message-passing structure in an FMS controller. Proceedings of the 1995 IEEE International Conference on Robotics and Automation, ICRA'95. Nagoya, Japan, 1447-54.

MMS (1990). Industrial Automation Systems - Manufacturing Message Specification. International Standard, ISO/IEC 9506-1, First edition.

Ramadge P. J. and W. M. Wonham (1987). Supervisory control of a class of discrete event processes. SIAM Journal of Control and Optimization, 25(1), 206-30.

Reinaldo Silva J., H. Afsarmanesh, D. D. Cowan and C. J. P. Lucena (1995). An object-oriented approach to the design of flexible manufacturing systems, in Balanced Automation Systems: Architectures and Design Methods, (eds. L. M. Camarinha-Matos and H. Afsarmanesh), Chapman \& Hall, U.K., 91-106.

Roshardt R., C. Uhrhan, T. Waefler and S. Weik (1995). A complimentary approach to flexible automation, in Balanced Automation Systems: Architectures and Design Methods, (eds. L. M. Camarinha-Matos and H. Afsarmanesh), Chapman \& Hall, U.K., 141-8.

Shlaer S. and S. J. Mellor (1992). Object Lifecycles - Modeling the World in States. Yourdon Press Computing Series, Prentice-Hall, U.S.A. 


\section{BIOGRAPHY}

Anders Adlemo received his M.Sc. in Electrical Engineering at Lund University of Technology, Lund, Sweden, in 1981, and Ph. D. in Computer Engineering at Chalmers University of Technology, Göteborg, Sweden, in 1993.

Dr. Adlemo is currently a researcher at the Department of Computer Engineering at Chalmers University of Technology. His current research interests include distributed computing systems, fault tolerant systems, and complex flexible manufacturing systems.

Sven-Arne Andréasson received his B.A. in Physics and Mathematics at the University of Göteborg, Sweden, in 1971, M.Sc. in Electrical Engineering at Chalmers University of Technology, Göteborg, Sweden, in 1976, and Ph. D. in Computer Science at Chalmers University of Technology, Göteborg, Sweden, in 1986.

Dr. Andréasson is currently an associate professor at the Department of Computing Science at Chalmers University of Technology. His current research interests include distributed computing systems, fault tolerant systems, databases, and complex flexible manufacturing systems.

Dr. Andréasson is a member of the Association for Computing Machinery (ACM), and the IEEE Computer Society.

Per Gullander received his M.Sc. in Automation Engineering at Chalmers University of Technology, Göteborg, Sweden, in 1992.

Mr. Gullander is currently a Ph.D. student at the Department of Production Engineering at Chalmers University of Technology. His current research interests include software tools for control system development, manufacturing system architectures and communication, and complex flexible manufacturing systems. 